\title{
CORRECTION
}

View Article Online

View Journal I View Issue

CrossMark

$<$ click for updates

Cite this: Phys. Chem. Chem. Phys., 2016, 18, 8752

DOI: $10.1039 / \mathrm{c} 6 \mathrm{cp} 90063 a$

www.rsc.org/pccp

\section{Correction: The thermodynamics of endosomal escape and DNA release from lipoplexes}

\author{
Yotam Y. Avital, ${ }^{\text {ab }}$ Niels Grønbech-Jensen ${ }^{\text {cd }}$ and Oded Farago*ab
}

Correction for 'The thermodynamics of endosomal escape and DNA release from lipoplexes' by Yotam Y. Avital et al., Phys. Chem. Chem. Phys., 2016, 18, 2591-2596.

The authors would like to correct an error in the published Fig. 2B, where the data for surfaces numbers 1 and 4 need to be interchanged. The amended figure and caption are shown below:
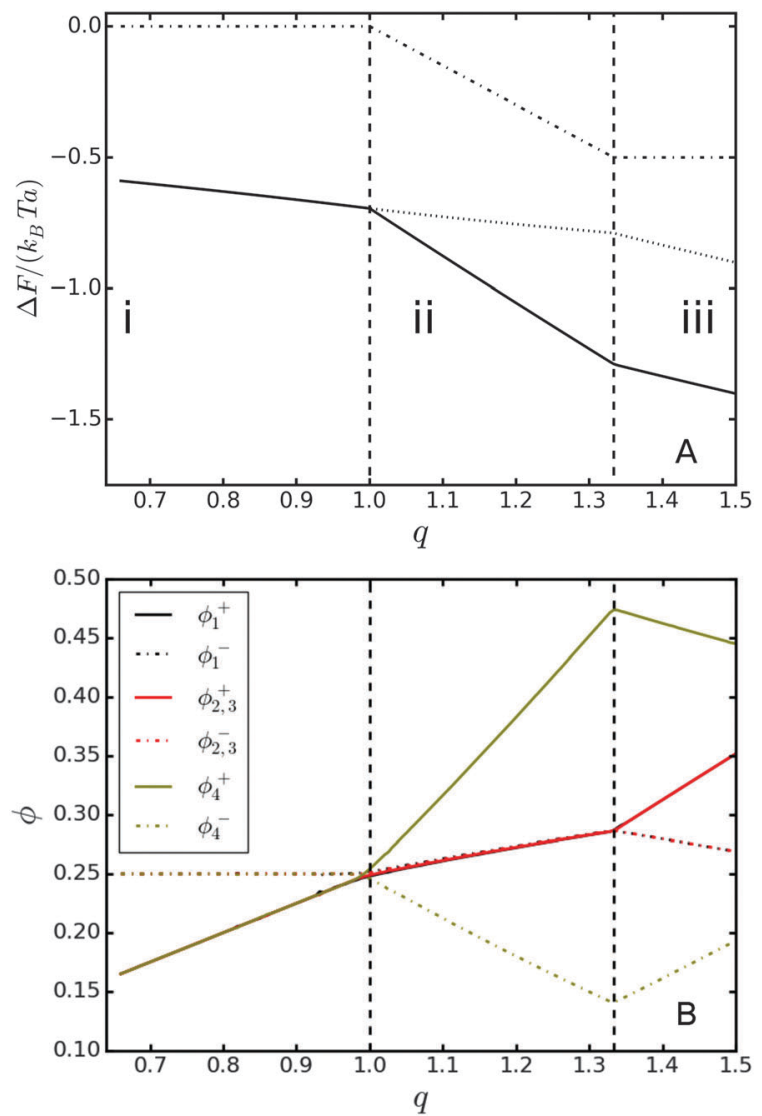

Fig. 2 (A) Solid line - the free energy $\Delta F$ (see text for definition), as a function of $q=2 \Phi_{+} / \Phi_{\mathrm{D}}$ with $\Phi_{\mathrm{D}}=1$ and $\Phi_{-}=0.5$. Dot-dashed and dotted lines show the partial contributions to $\Delta F$ originating, respectively, from counterion release and lipid mixing. (B) The equilibrium distribution of CLs (solid lines) and ALs (dot-dashed lines) in monolayers $i=1$ (black), $i=2,3$ (red) and $i=4$ (yellow). The vertical dashed lines mark the transition points between the different regimes discussed in the text.

The Royal Society of Chemistry apologises for these errors and any consequent inconvenience to authors and readers.

\footnotetext{
${ }^{a}$ Department of Biomedical Engineering, Ben-Gurion University of the Negev, Be'er Sheva 85105, Israel

${ }^{b}$ Ilae Katz Institute for Nanoscale Science and Technology, Ben-Gurion University of the Negev, Be'er Sheva 85105, Israel

${ }^{c}$ Department of Mechanical and Aerospace Engineering, University of California, Davis, California 95616, USA

${ }^{d}$ Department Mathematics, University of California, Davis, California 95616, USA
} 